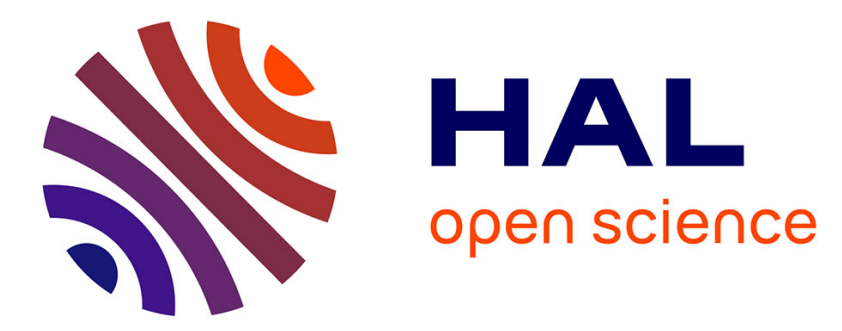

\title{
How do shape anisotropy and spatial orientation of the constituents affect the permittivity of dielectric heterostructures?
}

Christian Brosseau, Abderrahmane Beroual, Abdelmalek Boudida

\section{- To cite this version:}

Christian Brosseau, Abderrahmane Beroual, Abdelmalek Boudida. How do shape anisotropy and spatial orientation of the constituents affect the permittivity of dielectric heterostructures?. Journal of Applied Physics, 2000, 88 (12), pp.7278-7288. 10.1063/1.1321779 . hal-02148484

\section{HAL Id: hal-02148484 \\ https://hal.science/hal-02148484}

Submitted on 5 Jun 2019

HAL is a multi-disciplinary open access archive for the deposit and dissemination of scientific research documents, whether they are published or not. The documents may come from teaching and research institutions in France or abroad, or from public or private research centers.

$$
\text { Copyright }
$$

L'archive ouverte pluridisciplinaire HAL, est destinée au dépôt et à la diffusion de documents scientifiques de niveau recherche, publiés ou non, émanant des établissements d'enseignement et de recherche français ou étrangers, des laboratoires publics ou privés. 


\section{How do shape anisotropy and spatial orientation of the constituents affect the permittivity of dielectric heterostructures?}

Cite as: Journal of Applied Physics 88, 7278 (2000); https://doi.org/10.1063/1.1321779

Submitted: 15 December 1999 . Accepted: 06 September 2000 . Published Online: 28 November 2000

C. Brosseau, A. Beroual, and A. Boudida

\section{ARTICLES YOU MAY BE INTERESTED IN}

Effective dielectric constant of random composite materials

Journal of Applied Physics 81, 2375 (1997); https://doi.org/10.1063/1.364276

Permittivity of lossy composite materials

Journal of Applied Physics 83, 425 (1998); https://doi.org/10.1063/1.366725

A review and analysis of microwave absorption in polymer composites filled with carbonaceous particles

Journal of Applied Physics 111, 061301 (2012); https://doi.org/10.1063/1.3688435

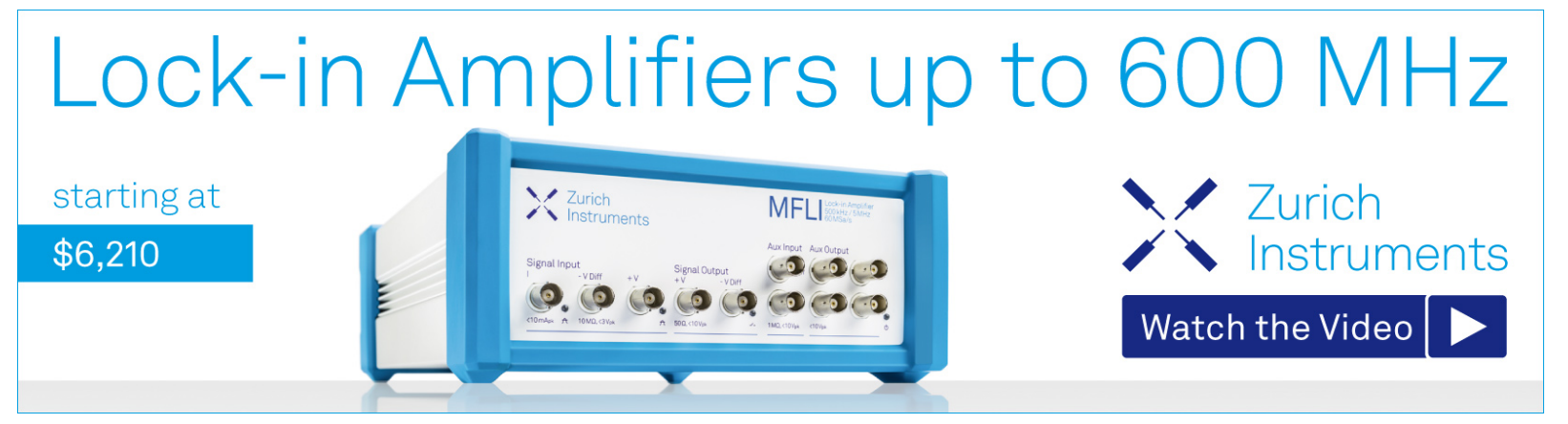




\title{
How do shape anisotropy and spatial orientation of the constituents affect the permittivity of dielectric heterostructures?
}

\author{
C. Brosseau ${ }^{\text {a) }}$ \\ Laboratoire d'Electronique et Systèmes de Télécommunications, Université de Bretagne Occidentale, \\ 6, avenue Le Gorgeu, B.P. 809, 29285 Brest Cedex, France
}

A. Beroual' ${ }^{\text {b) }}$ and A. Boudida

Centre de Génie Électrique de Lyon, École Centrale de Lyon, B.P. 163, 36 avenue Guy de Collongue, 69131 Ecully Cedex, France

(Received 15 December 1999; accepted for publication 6 September 2000)

\begin{abstract}
The study of dielectric heterostructures has been advancing at a rapid pace. Much of the interest in these materials stems from the fact that their physical properties can be systematically tuned by variation of the size and shape of the constituents. Here we report on extensive computer simulations of the effective permittivity of dielectric periodic (deterministic) heterostructures, having monosized hard core inclusions of anisotropic shape (rod, ellipsoid) embedded in an otherwise homogeneous and isotropic matrix. The real and imaginary parts of the permittivity, in the quasistatic limit, are rigorously evaluated with the use of the PHI3D field calculation package and the resolution of boundary integral equations. In this article, we show that the effective permittivity has critical properties near a conduction threshold. The conduction threshold concentration can be significantly modified by the size, shape, and spatial arrangement of the constituents. More specifically, it obeys a square law dependence as a function of the aspect ratio, i.e., the ratio of the smaller dimension to the larger dimension in both the rodlike and ellipsoidal inclusions. The data exhibit a scaling behavior and can all be collapsed onto a single master curve, indicative of a remarkable universality in the conductivity property. The critical exponents which determine how the real and imaginary parts of the effective permittivity scale with the distance from the conduction threshold are determined. Our results are compared with the scaling prediction of the standard percolation theory for infinite three-dimensional random lattices of insulator-normal metal composite systems. We also observed that the conduction transition is shifted towards higher concentrations as the angle between the symmetry axis and the direction of the applied electric field increases. Increasing the contrast ratio, between the permittivity and the conductivity of the background medium and the inclusions, results in dramatic changes of the complex effective permittivity, depending on the geometry of the inclusions. The scale-dependent properties and the mechanism which govern criticality are related to the actual area of contacts between the inclusions. (C) 2000 American Institute of Physics. [S0021-8979(00)07723-9]
\end{abstract}

\section{INTRODUCTION}

The answer to the title question is tremendously important for both academic research and industrial processes in a wide range of contexts from low to very high frequencies, e.g., electronics packaging and devices such as sensors, current limiters, acoustic actuators, and microwave absorbers. It has received recent attention with experimental and numerical findings that indicate that critical phenomena play a major role in the characterization of the complex permittivity of dielectric heterostructures. ${ }^{1-6}$ For such 1-3 composites, ${ }^{7}$ then, we have a rich picture that can be viewed from several perspectives: theoretical, numerical, and experimental. The objective of these studies is to provide a thorough understanding of the polarization mechanisms in a two-component

\footnotetext{
${ }^{a)}$ Electronic mail: brosseau@univ-brest.fr
}

${ }^{b}$ Electronic mail: beroual@trotek.ec-lyon.fr composite material consisting of monosized hard core inclusions of constituent 1 surrounded by a background material of constituent 2. A serious difficulty common to these studies is the detailed understanding of the underlying mechanisms responsible for the sharp increases in real and imaginary parts of the permittivity as the loading increases. Theoretical considerations do not give a unique answer to this question, but many reasonable suggestions have been proposed in the literature. , $^{2,8-10}$ The feature of this problem that we will address in a systematic and rigorous manner is the effect of the shape anisotropy of the inclusions on the permittivity of the composite material. We would like to begin reminding the reader of some results that are important to the discussion to follow. Although the systems considered in this study all have an ordered, periodic structure, we first examine several elements related to randomly disordered systems. One of the 
objectives of this work will be to put in parallel percolation on a random structure and close packing for a regular periodic structure.

\section{A. Critical behavior}

Much progress has been achieved in the study of the dielectric behavior in heterostructures by the infusion of percolation ideas from statistical physics and critical phenomena. The achievement of a percolation mechanism in condensed matter materials has focused a large research effort within the physics community owing to its wide range of applications. ${ }^{1,4}$ Useful references which provide an introduction to the basic principles of percolation are several articles ${ }^{6}$ and the books by Stauffer and Aharony ${ }^{5}$ and Sahimi. ${ }^{11}$ Insulator-normal metal (random) composite systems are the classic and best studied examples. For these systems, the conductivity exhibits generic properties as the concentration of metallic component is increased from the pure insulator state. ${ }^{5}$ Percolation manifests itself in the sharp increase of the measured dc conductivity which follows a power-law dependence in the close proximity to the percolation threshold characterized by a volume fraction $f_{c}$. Percolation theory predicts that the dc electrical conductivity $\sigma$ obeys a scaling relation $\sigma \sim\left(f-f_{c}\right)^{t}$, as $f$, is approached from the conducting side $\left(f>f_{c}\right)$ where $f$ denotes the volume fraction of the high conductivity component, and $\sigma \sim\left(f_{c}-f\right)^{-s}$, where $s$ describes the divergent behavior of conductivity, when $f$, is approached from the insulating side $\left(f<f_{c}\right)$. Similarly, the real part of the permittivity shows a critical behavior, on both sides of $f_{c}, \varepsilon^{\prime} \sim\left|f-f_{c}\right|^{-s}$. Theory then dictates that the dielectrical behavior at percolation threshold must be associated with critical exponents like $t$ and $s$ which depend only on the spatial dimension, i.e., for three-dimensional systems $t \cong 1.5-2.0$ and $s \cong 0.7$, and not on the lattice or percolation type. $^{4,5,11}$ It seems reasonable to suggest that some of the ideas developed for percolation models in insulator-normal metal mixtures may, in fact, be useful in understanding the threshold conduction occurring in dielectric heterostructures. Whether or not the mechanism(s) leading to the sharp increase evidenced in dielectric-dielectric mixtures under certain circumstances differ from that of insulator-normal metal mixtures needs to be clarified. In this article, we study periodic structures for which the threshold concentration corresponds to the close packing of inclusions and can be derived by simple geometrical treatment. Whether the rapid rise in permittivity arising at, or near contact, has a critical behavior like the percolation threshold of random composites we just discussed, and if so whether a power-law description will be operative are questions not yet decided.

\section{B. Experimental studies and scaling behavior}

Although a considerable number of experimental studies have been recorded in which the electrical conductivity and permittivity of composites with spherical inclusions have been considered, far fewer studies have been concerned with the dielectric behavior of anisotropic structures. Recently, a number of authors have entertained the possibility that fiber inclusions in polymeric matrices are of practical importance to investigate this problem. ${ }^{12-19}$ Interestingly, the importance of a proper treatment of the aspect ratio contribution to the dielectric behavior of carbon fiber composites, by controlling (rheologically and magnetically) the fiber orientations and the aggregation in the specimens, was emphasized by Sturman and McCullough, ${ }^{12}$ and Carmona et al. ${ }^{13}$ These authors emphasized the difficulty of accurately assigning the experimental value of the conduction threshold. In the past, there have been several experimental searches ${ }^{14-16}$ to see how $f_{c}$ scales with $r / l$, where $r$ and $l$ are respectively the radius and the length of the rodlike inclusion. Experiments on fiber composites have not yet been able to resolve the disputes between theories and simulations. ${ }^{20}$ In particular, we shall refer later to work by Carmona et al. ${ }^{13}$ and Balberg et al. ${ }^{16}$ Carmona et al. have conducted an extensive study of the electrical conductivity of composites made of carbon fibers with various radius-to-length ratios blended with epoxies and silicon elastomers as host polymers. ${ }^{13}$ To analyze their data, Carmona et al. applied heuristic dimensional arguments which amount to being able to express the dc conductivity $\sigma$ in terms of the scaling form $l^{2} \sigma=F\left(l^{2} f\right)$, where $f$ refers to the volume fraction of fibers. However their experiments spanned a narrow range of $l(1$ to $3 \mathrm{~mm})$ and the range of $f$ over which the scaling form holds was not explored, i.e., $r / l$ small enough and/or $f$ not too large. Additionally, Balberg and co-workers have suggested excluded volume constraints to explain the dependence $\sim(r / l)^{2}$ of the percolation threshold. ${ }^{16}$ In another study, Carmona and El Amarti reported conductivity measurements on graphite fiber reinforced polymers. ${ }^{17}$ These experimental measurements further suggested that the percolation transition may belong to a new universality as the anomalously large critical exponent of the conductivity seen in their experiments cannot be reconciled with the universal value. ${ }^{17}$ An interesting feature of the work by Lagarkov and collaborators ${ }^{14}$ is the observation by these authors that the square law dependence, i.e., $f_{c} \sim(r / l)^{2}$ can be derived by a standard effective medium approximation. Interestingly, these authors renormalized the depolarization factor in the vicinity of the percolation threshold concentration and found a $r / l$ dependence of $f_{c}$, which represents a substantial departure from the phenomenology of effective medium theories. However, it is not clear what is the maximal $r / l$ ratio up to which the percolation threshold concentration can be described by a $r / l$ law. ${ }^{13,15,20-22}$ Moreover, our ability to interpret the existing and new experiments is limited by the imprecision in our knowledge of the relevant critical exponents. It should be also noted that the variation in the rod length distribution and the presence of small quantities of impurities which can act as chemical cross links may lead to dramatic changes in the value of $f_{c}$. The above studies concern real (random) composite materials. We are not aware of similar investigations for periodic composite materials.

\section{Modeling of effective permittivity}

The effective permittivity of heterostructures is a rather old problem and has a venerable history. ${ }^{10}$ A number of analytic approaches, based on mixing rules and bounds, have been used to analyze the permittivity for a medium in which 
one of the components can be considered as a host in which inclusions, assumed to have a convenient shape (usually spherical or ellispoidal), of the other components are embedded. ${ }^{1}$ Many mixing laws have been formulated with a specific problem in mind to describe experimental observations, often couched in terms of empirically determined parameters. The great advantage of these mixing laws stems from the possibility of tailoring them over a wide range of conditions. But it should be mentioned that despite their obvious merits and attractive features for variety of experiments and practical applications, mixing laws suffer from different shortcomings. These shortcomings have long been identified as originating from the missing multipole interactions, but attempts to overcome these failures have been limited to relatively simple situations, such as the dilute limit, or the quasistatic approximation. Another aspect of the difficulty is that mixing laws cannot describe the substantial changes in dielectric properties in the vicinity of the percolation threshold. Phenomenological analysis based on effective-medium approximation (EMA) have been also studied in the literature. EMA is a self-consistent method of determining the effective properties of a heterogeneous medium, in which the medium is replaced with a hypothetical (uniform) effective medium whose properties are determined self-consistently. ${ }^{1,10}$

There has been considerable progress in recent years towards the development of high-accuracy first-principles models for computational electromagnetics. Computational electromagnetics, a field at the intersection of computer science and electromagnetics, is intimately bound to diverse subdisciplines within physical science. As the sophistication of modern numerical techniques grows, materials scientists have started to seek an understanding and control of permittivity that extends beyond mere heuristic descriptions, e.g., mixing laws. Ab initio calculations have become an increasingly important source of fitting data. ${ }^{2,3,23-29}$ However, the computational difficulty of properly describing the internal (random) microstructure has so far prevented an accurate determination of the local electric field distribution in realistic (real-world) composite materials. ${ }^{30,31}$ At present, exact simulations are possible only for highly simplified models, i.e., periodic heterostructures, a situation common to studies of many complex systems. This renders the problem deterministic and, therefore, amenable to a unique solution. During the past 20 years or so, the development of efficient algorithms to carry out such calculations has been an active area of research. Motivated by these developments, some time ago we began to numerically investigate the combined effects of finite conductivity and shape anisotropy on the calculation of the complex permittivity of two-component dielectric heterostructures. ${ }^{24-26}$ The permittivity is determined by a number of factors, such as the volume fraction and the permittivity of the constituents, the spatial arrangement, and the size distribution of inclusions. The algorithm solves the Laplace equation by converting boundary integral equations into a sparse eigenvalue problem. An important ingredient in our is that these $a b$ initio calculations rely on the use of the PHI3D field calculation package. We performed a large number of checks on various two- and three-dimensional simple microstructure geometries which serve also on the applicability and utility of this modeling technique of more complicated systems. ${ }^{24-26,32}$

\section{Purpose of the present work and outline}

This representative but far from exhaustive literature survey highlights the considerable interest and importance that has been attached to the answer to the title question. The present study represents a natural continuation of a previous paper $^{3}$ to assess the relative importance of factors suggested in prior experiments and models as affecting the effective complex permittivity. But our real motivation in presenting these results is to investigate in detail the influence of shape anisotropy (rod and ellipsoid) and spatial orientation on the rapid change of the real and imaginary parts of the permittivity near the conduction threshold. The current work was also undertaken in support of experimental efforts in this body of literature.

With these considerations in mind, the outline of the remainder of this paper is as follows: we first provide in Sec. II the relevant background including a brief overview of the model and the numerical calculations. Effective permittivity calculations are presented in Sec. III in terms of shape anisotropy, conductivity, and spatial orientation of the inclusions: the numerical results are compared with the prediction of the percolation theory. Section IV discusses the significance of these results. Finally, in the concluding remarks, Sec. IV, we suggest future directions for continuing the development of these simulations.

\section{BACKGROUND AND NUMERICAL PROCEDURE}

The guiding principles of the general algorithm used here for computing the effective permittivity of dielectric heterostructures will be given in this section along with some necessary background. Full details have been given elsewhere ${ }^{24-26,32}$ and we recount here only the salient points.

\section{A. Background information}

In Refs. 24-26 and 32, the physics of the problem was expressed mathematically by a boundary value problem for the electrostatic potential in the material medium. At the outset, we make a number of basic ingredients in these calculations. The first is to consider that the medium consists of homogeneous elements of two constituents, say 1 for the inclusions and 2 for the host matrix, where the local permittivity varies from constituent to constituent but has a fixed value in a given constituent. The whole medium is placed between the flat parallel plates of a capacitor. The second is the quasistatic approximation: the scale of inhomogeneities in the actual heterostructure is small compared to the relevant scales within the material, e.g., wavelength and skin depth. Thus, we treat the material medium as a homogeneous equivalent material. The effective (relative) permittivity $\varepsilon$ can be defined as $\bar{E}^{2} \varepsilon=(1 / \Omega) \int_{\Omega} \hat{\varepsilon}|\nabla V|^{2} d \Omega$ where $\hat{\varepsilon}$ is the local permittivity value, $\bar{E}=(1 / \Omega) \int_{\Omega} E d \Omega$ denotes the average field which depends on the applied potentials, $V$ is the potential, $E$ is the local electric field in the cell, and $\Omega$ is the volume of the medium. Note that this definition ensures that 
if the composite medium were replaced by a homogeneous medium, subject to the same boundary conditions, the rate of dissipation would be the same. The third is that higher electric multipole interactions are treated exactly. This is of fundamental importance because higher multipole interactions become important when the inclusions approach contact. This method goes beyond the mean field approximation which is at the basis of many effective medium laws.

\section{B. Numerical method and code}

The basic computational approach that is employed in this study is similar to that used by some of us in previous investigations. ${ }^{24-26,32}$ It should be noted that a number of modeling techniques have been used by other researchers, during the last decade, to investigate the dielectric properties of heterostructures. ${ }^{22-29}$ In the difficult task of developing numerical codes it has been found useful to adopt the boundary-element code PHI3D to perform the numerical analysis. Previous work in our group has established that this modeling procedure is a viable and versatile technique to perform the computations. ${ }^{24-26,32}$

Since details regarding the formulating numerical procedures may be found in earlier publications, only a brief overview is given here. The basic elements of our simulations are carried out as follows. In these calculations, the starting point is the Laplace equation, i.e., $\nabla(\varepsilon \nabla V)=0$ for given geometry and boundary conditions, i.e., $\nabla V \cdot \mathbf{n}=0$ at the boundaries, where $\mathbf{n}$ is the local unit vector normal to the surface. A constant electric field is imposed and is assumed to be, without loss of generality, parallel to the $z$ axis. The calculation depends on the shape, the permittivity, and the orientation of the inclusions in the matrix, assuming that all of the inclusions have a hard core. In the first step, the necessary boundary integral equations required to calculate the electrostatic potential distribution for the material medium are formulated. Then, the integral equations are converted to matrix equations. Next, to solve these matrix equations, we use a matrix inverse iteration approach based on the Galerkin method of weighted residuals. One salient feature of our method is that only the boundaries of the geometry need be discretized, not the entire volume, which has the effect to reduce the memory space required for manipulation of data, but the matrix equation to solve is asymmetric and full. The PHI3D field calculation package considers a semi-automatic meshing system. In simple test cases, this approach has duly led to the same results as the ones obtained from the application of other numerical methods. ${ }^{24-26}$ The program uses a general curvilinear grid to divide the computational domains into discrete cells and integrate the governing equations on the individual cells to construct algebraic equations for the discrete unknowns. Figures 1(a)-1(c) illustrate the finiteelement mesh for each unit (cubic) cell considered in the present work. Two things are worth observing: on the one hand, the symmetry of the structures provides that we only need to simulate the potential distribution over $1 / 8$ th of one inclusion, and on the other hand, the results were found the same if the elementary cells shown in Fig. 1 were parallepipeds rather than cubes. ${ }^{24}$ Note that, formally, the inclusions

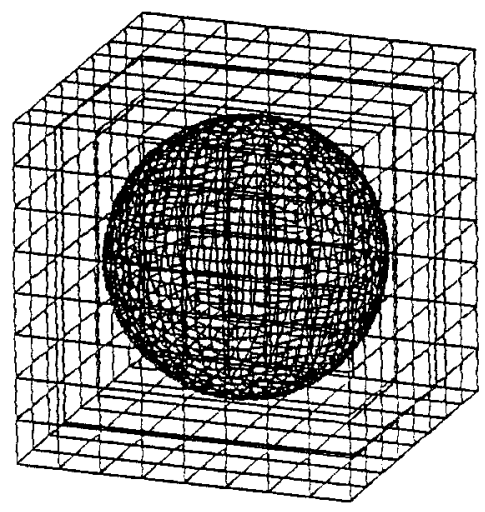

(a)

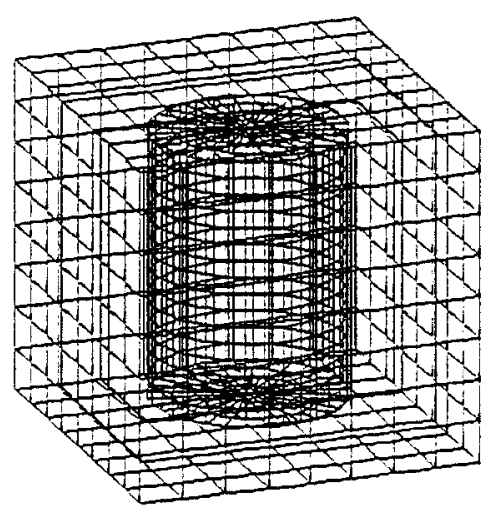

(b)

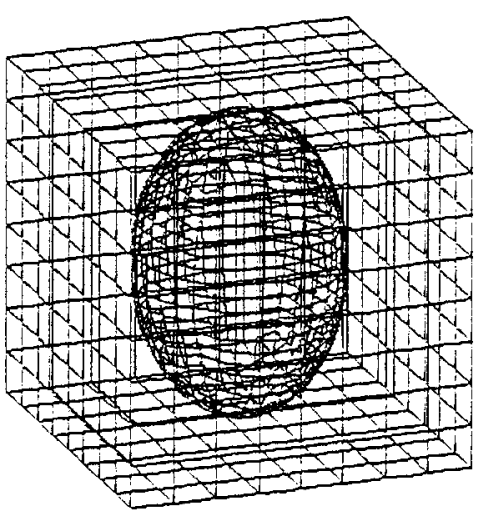

(c)

FIG. 1. Finite-element mesh for the unit cells used for the numerical analysis: (a) spherical inclusion, (b) rodlike inclusion, and (c) ellipsoidal inclusion.

are allowed to fuse each other, i.e., the region of permittivity $\varepsilon_{1}$ can intercept the sides of the parallelipipedic cell. ${ }^{2,24,32}$ The computational requirements of this code are handled easily by the random access memory and speed of large modern workstations. The run time for a typical calculation ranges from a few minutes to a few hours for calculating the permittivity of a typical three-dimensional configuration on a HP model 712/80 workstation. As was initially worked out in detail in Refs. 24-26 and 30, this simulation method has the advantage of having a low computational cost.

\section{SIMULATION RESULTS}

We present here three detailed results, to illustrate the effects of shape anisotropy, spatial orientation, and conductivity of the inclusions on the effective permittivity, we have 
achieved using the numerical procedure described in Sec. II. In the following subsections, the $a b$ initio simulations were conducted on periodic heterostructures having a simple cubic lattice. A detailed comparison between anisotropic and spherical inclusions is provided. We defer to Sec. IV any discussion of these results.

\section{A. Effect of shape anisotropy}

As an illustrative example, all the calculations presented in this subsection were carried out with the values $\varepsilon_{1}=80$ $-i 10^{2}$ and $\varepsilon_{2}=2-i 0$. We have selected as a test case this set of values because of potential applications to biological systems. ${ }^{18}$

\section{Rodlike inclusions}

The first series of calculations were performed on rodlike inclusions. More specificaly, the rod radius is kept fixed and the volume fraction of the inclusions changes by varying the rod length $l$. In our calculations, we varied the rod radius $r$ from 0.05 to 0.45 . The details of numerical results for rods have been described elsewhere, ${ }^{3}$ so only a short summary is given here. In Figs. 2(a) and 2(b), the real and imaginary parts of the relative complex permittivity are plotted versus the volume fraction $f$, respectively. The most distinguishing feature of the data is the sharp increase in permittivity for $f \equiv f^{*}$, which is particularly evident in the imaginary part. This "conduction threshold" marks the transition from insulating to conducting behavior and is identified with the asymptotic variations of $\varepsilon^{\prime}$ and $\varepsilon^{\prime \prime}$ near $f^{*}$. We found that the conduction threshold shifts to a greater specimen radius as the rod fraction increases, i.e., $f^{*} \sim r^{2}$ (Fig. 2 of Ref. 3). Observe that the quadratic law corresponds to the close packing condition since $f^{*}$ occurs when the cylinders touch. For this system in which one cylinder occupies each unit cell, with $L$ the side length of the unit cell, the volume fraction of inclusion has the form $f=\pi r^{2} l / L^{3}$. For cylinders arranged on a simple cubic lattice, the close packing condition occurs for $l=L$ if the radius is kept fixed. Then $f^{*}$ is given by $f^{*}$ $=\pi(r / l)^{2}$. It is useful to compare the permittivity calculated for a composite based on rodlike and spherical inclusions. Comparing these graphs in Figs. 2(a) and 2(b), one can observe that the volume fraction dependence of the permittivity is strikingly different for rodlike inclusions from its behavior for spherical inclusions. Another relevant feature of our recent simulation results is that $\varepsilon^{\prime \prime}$ is related to $f$ through the heretofore unobserved scaling behavior $\varepsilon^{\prime \prime}=r F\left(f / f^{*}\right)$ : all the data for the imaginary part of the permittivity can be scaled onto a single master curve. ${ }^{3}$ This scaling demonstrates that, despite the wide variation in the aspect ratio, a single parameter controls the dielectrical behavior of these composites. As of yet, the question concerning the functional form of $F(x)$ is not settled. Of interest is the evolution of the power-law fits applied to the real and imaginary parts of the permittivity in the range $10^{-3}<\left|f-f^{*}\right|<10^{-1}$. Figures 3(a) and 3 (b) show $\varepsilon^{\prime}$ and $\varepsilon^{\prime \prime}$ as a function of the distance from the conduction threshold, $f-f^{*}$, in a double-logarithmic scale. Linear fits to the $\log -\log$ plots of $\varepsilon^{\prime}$ and $\varepsilon^{\prime \prime}$ give scaling laws which can be characterized by two critical exponents $s^{*}$ and $t^{*}$ according to $\varepsilon^{\prime} \sim\left(f^{*}-f\right)^{-s^{*}}$, and $\varepsilon^{\prime \prime}$
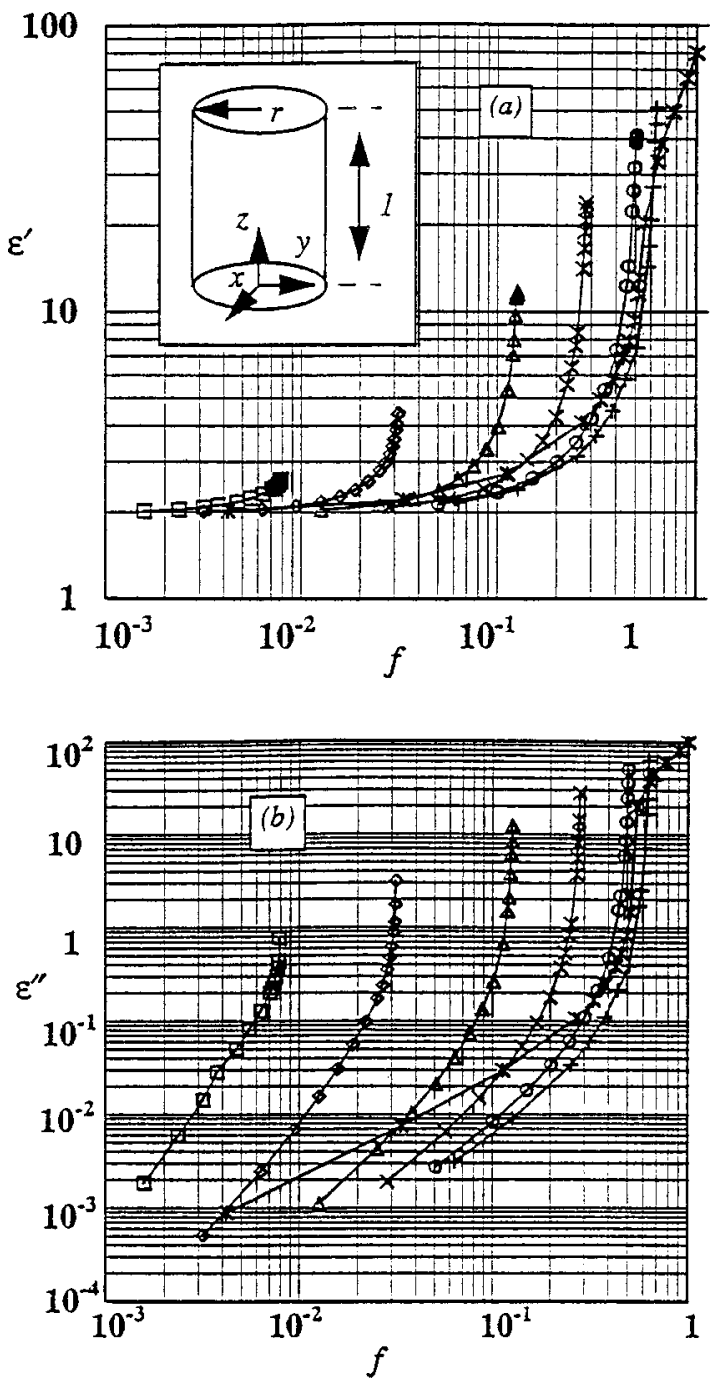

FIG. 2. (a) Real part of the effective permittivity $\varepsilon^{\prime}$ is shown, in a $\log -\log$ plot, as a function of the volume fraction $f$ of the inclusion phase. Rodlike inclusions (permittivity $\varepsilon_{1}=80-i 10^{2}$ ) are placed in a host matrix material of permittivity $\varepsilon_{2}=2-i 0$. Simple-cubic lattice configuration. Inset shows the configuration of the two-component periodic composite material investigated in this study. Symbols are: $(\square) r=0.05,(\diamond) r=0.10,(\triangle) r=0.20$, $(\times) r=0.30,(\bigcirc) r=0.40$, and $(+) r=0.45$. The solid lines are guides to the eye. The case of spherical inclusions is also shown $(*)$. The direction of the applied electric field is parallel to the rod axis. (b) Same as in (a) for the imaginary part of the effective permittivity $\varepsilon^{\prime \prime}$. Inset indicates how the volume conduction threshold concentration $f^{*}$ is defined.

$\sim\left(f-f^{*}\right)^{t^{*}}$ with $s^{*}=0.66 \pm 0.10$ and $t^{*}=1.30 \pm 0.19$ as parameters. Note that these values of $s^{*}$ and $t^{*}$ do not coincidate either with the universal values of the standard percolation model, or with the mean field exponents calculated by several authors. ${ }^{33}$ We had earlier noted the values $s^{*}=0.65$ \pm 0.15 and $t^{*}=0.80 \pm 0.05$ for spherical inclusions. ${ }^{2}$

\section{Ellipsoids}

We now use simulation data to quantify variations of effective permittivity for ellipsoidal inclusions. To the best of our knowledge, we do not know of any published work on the critical aspects of ellipsoid composite electrical behavior. Figures 4(a) and 4(b) show the real and imaginary components of the composite material with the long axis of the 

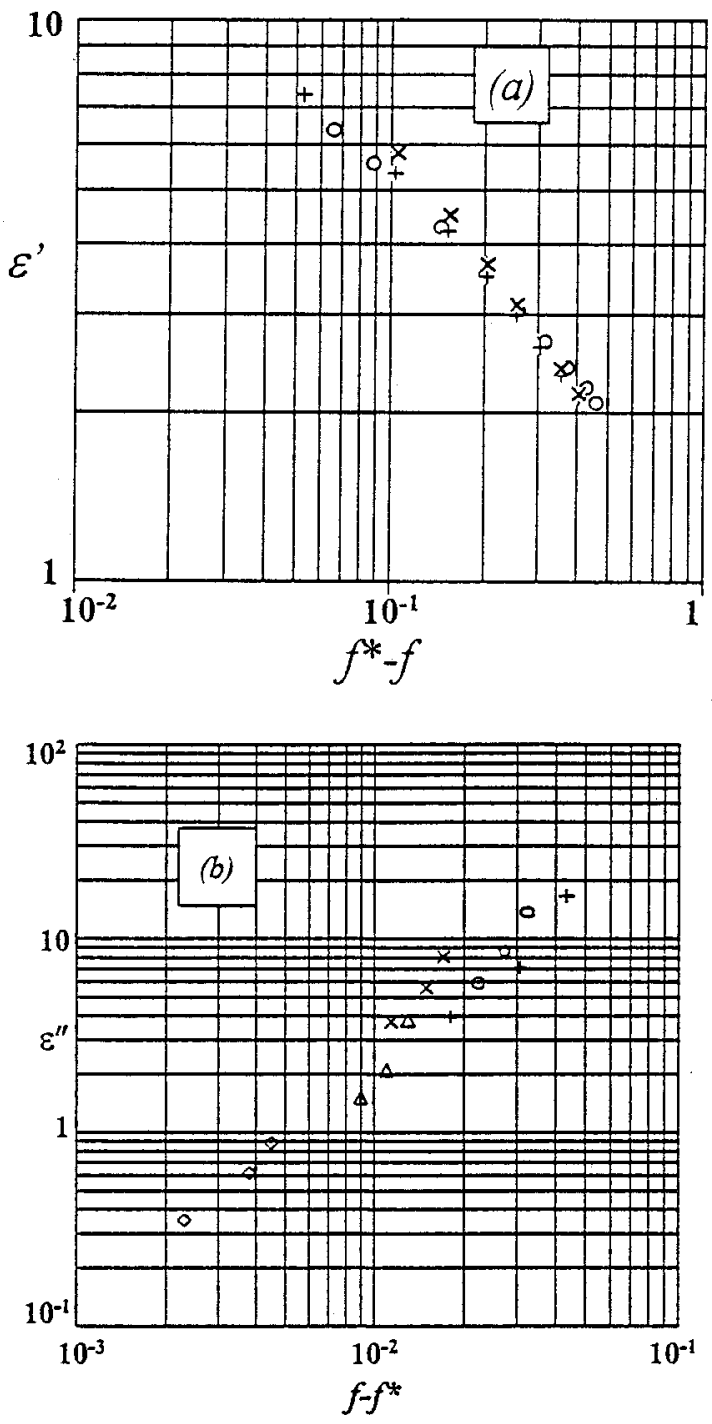

FIG. 3. (a) Real part of the permittivity $\varepsilon^{\prime}$ as a function of the scaling variable $f-f^{*}$, where $f<f^{*}$. In a $\log -\log$ plot the points fall on a straight line with slope $s^{*}=0.66 \pm 0.10$. Same symbols as in Fig. 2(a). (b) Same as in (a) for the imaginary part of the effective permittivity $\varepsilon^{\prime \prime}$, where $f>f^{*}$. In a $\log -\log$ plot the points fall on a straight line with slope $t^{*}=1.30$ \pm 0.19 . Same symbols as in Fig. 2(a).

inclusion parallel to the direction of the applied uniform electric field, and where $c$ is the length of the principal symmetry axis and $\mathrm{a}$ is the length of each axis perpendicular to the principal symmetry axis. Here we assume $c>a=b$ which describes a prolate spheroid. Figures 4(a) and 4(b) reveal the following things. First, at very low loading of inclusions, the composite permittivity remains at the level of the permittivity of the unfilled matrix, $\varepsilon_{2}$. As the inclusion loading is increased, in a certain region the permittivity increases rapidly by many orders of magnitude. Second, this sharp nonlinear variation in $\varepsilon^{\prime}$ and $\varepsilon^{\prime \prime}$ is sensitive to the aspect ratio (short axis length/long axis length) of the inclusions. However, despite their differences all the data do have some features in common. For instance, we have analyzed the scaling of the conduction threshold concentration with the length $a$. Specifically, our data show a square law dependence, i.e., $f^{*} \sim a^{2}$, as can be deduced from Fig. 5. One can see that this result is consistent with the close packing con-
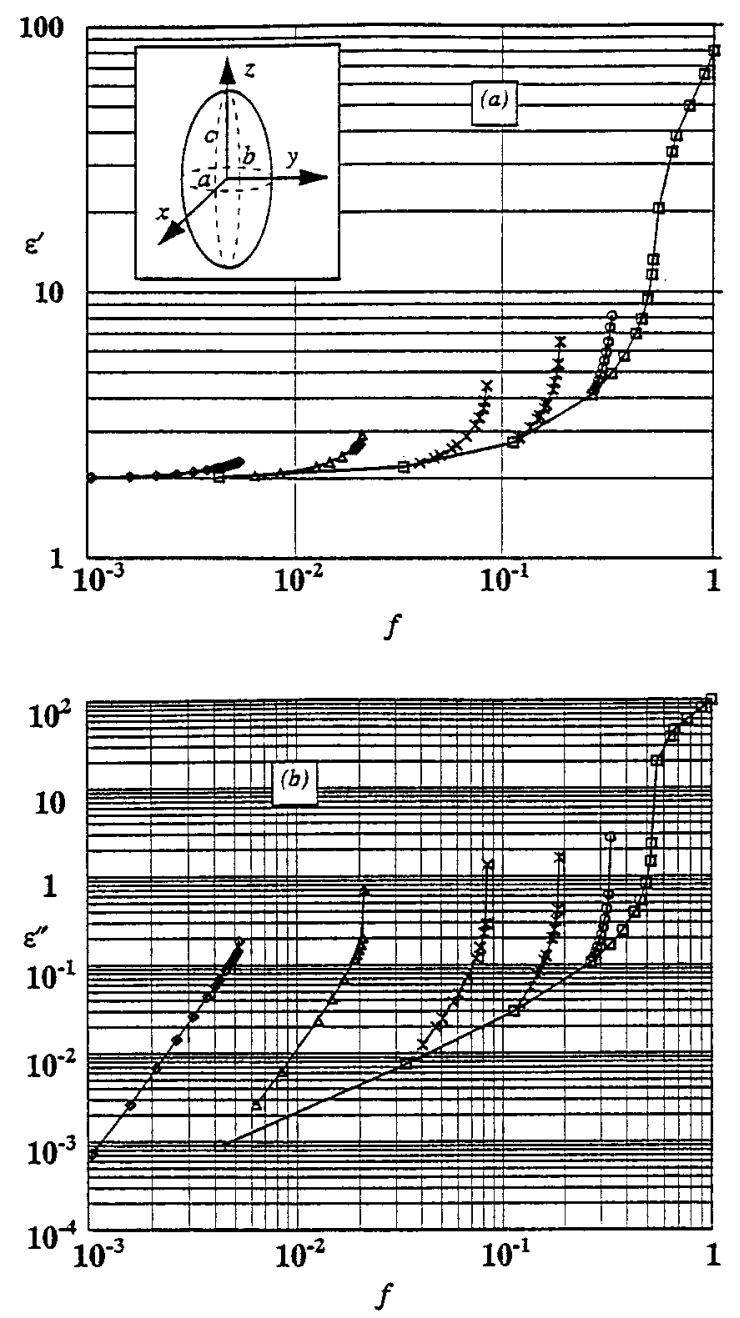

FIG. 4. Real part of the effective permittivity $\varepsilon^{\prime}$ is shown, in a $\log -\log$ plot, as a function of the volume fraction $f$ of the inclusion phase. Ellipsoidal inclusions (permittivity $\varepsilon_{1}=80-i 10^{2}$ ) are placed in a host matrix material of permittivity $\left(\varepsilon_{2}=2-i 0\right)$. Simple-cubic lattice configuration. Inset schematically shows the configuration of the two-component periodic composite material investigated in this study. Symbols are: $(\diamond) a=b=0.05,(\triangle) a$ $=b=0.10,(\times) a=b=0.20,\left({ }^{*}\right) a=b=0.30$, and $(\bigcirc) a=b=0.40$. The solid lines are guides to the eye. The case of spherical inclusions is also shown $(*)$. The direction of the applied electric field is parallel to the principal symmetry axis. (b) Same as in (a) for the imaginary part of the effective permittivity $\varepsilon^{\prime \prime}$.

dition for a system in which one prolate spheroidal inclusion occupies each unit cell of side length $L$. This is a direct analogue of the above result for cylinders. Here, the volume fraction of inclusion is $f=4 \pi a^{2} c / 3 L^{3}$. For prolate spheroids arranged on a simple cubic lattice, the close packing condition occurs when $c=L / 2$, corresponding to $f^{*}=(\pi / 6)$ $\times(a / c)^{2}$. The data exhibit an unexpected scaling behavior (see Fig. 6) that transcend the large variations: all the data can be collapsed onto a single master curve, $\varepsilon^{\prime \prime}$ $=a F\left(f / f^{*}\right)$, indicating of a universality in the conductivity property. To analyze the critical behavior more quantitatively, we find that it is convenient to use the power-law analysis. The results are shown to scale onto single curves like Figs. 3(a) and 3(b). As the critical point is approached, the numerical data and a least-square fitting provides the exponents $s^{*}=0.41 \pm 0.07$ and $t^{*}=0.57 \pm 0.04$. We observe 


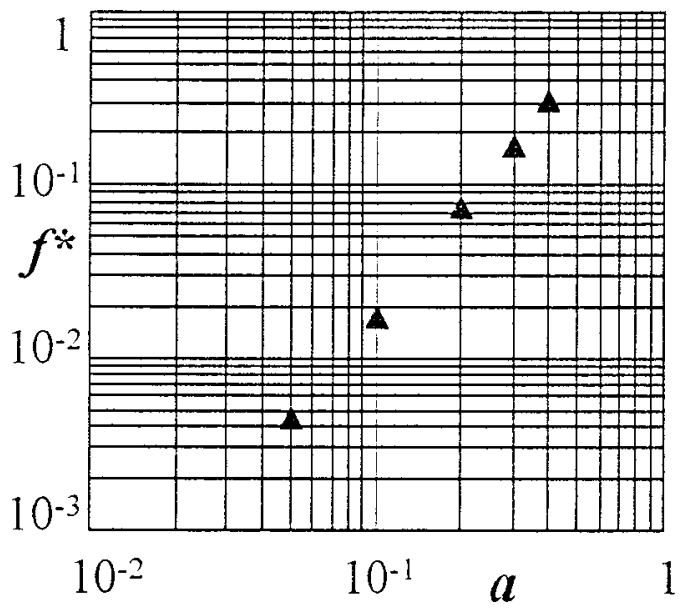

FIG. 5. Conduction volume threshold concentration $f^{*}$ as a function of the length of the semi-minor axis of the ellipsoid, $a$.

that they are different from the values obtained for rodlike inclusions (see Table I for comparative purpose). We will return to a discussion of these values later.

\section{B. Effect of spatial orientation}

The next important step is to determine how spatial orientation of the inclusions affect the permittivity of the material. All the calculations presented in this section are based on the values $\varepsilon_{1}=80-i 10^{2}$ and $\varepsilon_{2}=2-i 0$.

\section{Rodlike inclusions}

We made extensive calculations of the angular dependence of the permittivity. In Figs. 7(a) and 7(b), we present a plot of $\varepsilon^{\prime}$ and $\varepsilon^{\prime \prime}$, as a function of the volume loading for several values of the angle of the symmetry axis of the rod $(r=0.10)$ relative to the direction of the applied electric field, $\alpha$. A more revealing way to present these results is to plot these quantities as a function of $\alpha$ for two values of $f$, e.g., $f=0.05$ and $f=0.113$. The inset of Fig. $7(\mathrm{~b})$ shows such representation for $\varepsilon^{\prime \prime}$. The same trend (not represented) is observed for $\varepsilon^{\prime}$. As the value of $\alpha$ approaches $90^{\circ}$, i.e., the

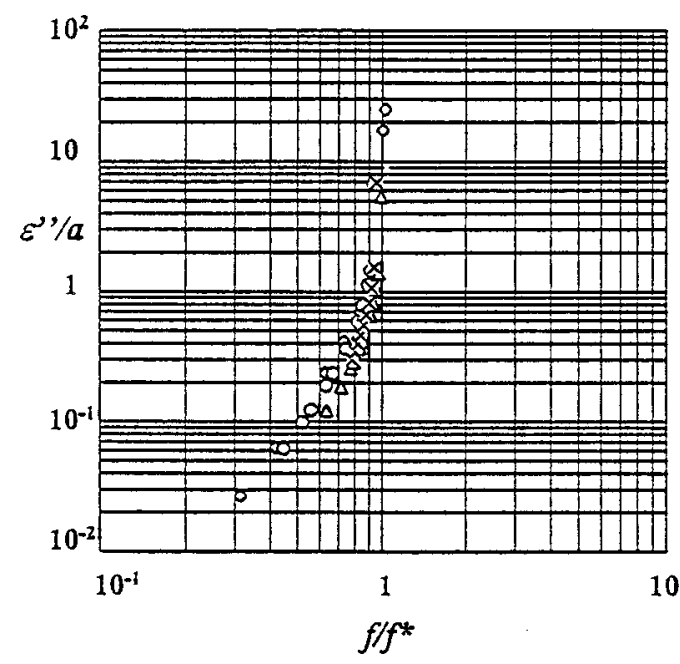

FIG. 6. Imaginary part of the effective permittivity $\varepsilon^{\prime \prime}$ as a function of the normalized volume concentration $f / f^{*}$. Same symbols as in Fig. 4(a).
TABLE I. Results of fitting the real and imaginary parts of the effective permittivity of two-component composite materials to a simple power law giving the critical exponents $s^{*}$ and $t^{*}$. Inclusions of permittivity $\varepsilon_{1}=80$ $-i 10^{2}$ are placed in a host matrix of permittivity $\varepsilon_{2}=2-i 0$. Simple-cubic lattice configuration.

\begin{tabular}{lcc}
\hline \hline Inclusion & \multicolumn{1}{c}{$s^{*}$} & $t^{*}$ \\
\hline Sphere & $0.65 \pm 0.15$ & $0.80 \pm 0.05$ \\
Rod & $0.66 \pm 0.10$ & $1.30 \pm 0.19$ \\
Ellipsoid & $0.41 \pm 0.07$ & $0.57 \pm 0.04$ \\
\hline \hline
\end{tabular}

condition most defavorable for a large area of contact, $\varepsilon^{\prime}$ and $\varepsilon^{\prime \prime}$ decrease significantly. Note that we were not able to determine $f^{*}\left(\alpha>0^{\circ}\right)$, and consequently, the $s^{*}$ and $t^{*}$ values, because of computational limitation: what we can say is that the values of $f^{*}\left(\alpha>0^{\circ}\right)$ are higher than $f^{*}\left(\alpha=0^{\circ}\right)$. This result is very similar to what Agari et al. had recently reported in a study of polyethylene composites filled with oriented carbon fibers. ${ }^{34}$
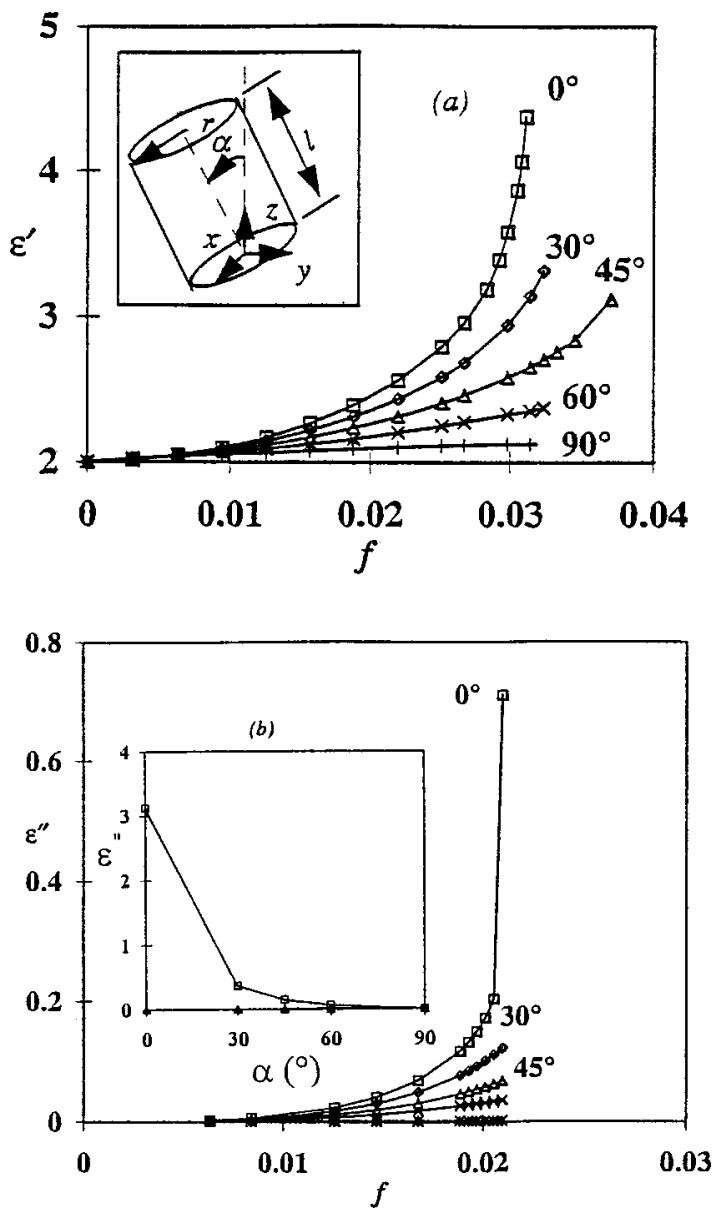

FIG. 7. (a) Same as in Fig. 2(a) for the real part of the effective permittivity when the rod axis is oriented at an angle $\alpha$ with respect to the electric field vector direction, i.e., the $z$ direction, see the inset for notations. Symbols are: $(\square) \alpha=0^{\circ},(\diamond) \alpha=30^{\circ},(\triangle) \alpha=45^{\circ},(\mathrm{x}) \alpha=60^{\circ}$, and $(+) \alpha=90^{\circ}$. Here $r=0.10$. (b) Same as in (a) for the imaginary part of the effective permittivity. The inset shows the angular dependency of $\varepsilon^{\prime \prime}$ when $f=0.05(\triangle)$ and $f=0.113(\square)$. 


\section{Ellipsoids}

Similar to the angular dependencies shown in Figs. 7(a) and 7(b) the same trend for ellipsoidal inclusions is found [the figures are omitted because of space limitations]: this is a clear manifestation of a strong shape anisotropy effect on the dielectric characteristics of heterostructures. In like fashion, with increasing $\alpha$, we observe that $\varepsilon^{\prime}$ and $\varepsilon^{\prime \prime}$ decrease.

\section{Effect of conductivity}

It is now time to determine to what extent large values of $\varepsilon_{1}^{\prime}$ and $\varepsilon_{1}^{\prime \prime}$ affect the permittivity of the composite material. All the calculations presented in this section are based on a wide selection of the real and imaginary parts of $\varepsilon_{1}=\varepsilon_{1}^{\prime}$ $-i \varepsilon_{1}^{\prime \prime}$, i.e., $\varepsilon_{1}^{\prime}$ ranges from 5.5 to 30 , and $\varepsilon_{1}^{\prime \prime}$ ranges from $10^{-2}$ to 0.5 , and $\varepsilon_{2}=2.2-i 10^{-4}$. We have selected as test cases these sets of values because of potential applications to high-energy density capacitors, particularly for space-power technology.$^{35}$ For example, composites which have large real part of permittivity and low imaginary part of permittivity with low volume concentration of inclusions would be desirable for their lower weight and lower cost.

\section{Rodlike inclusions}

For comparative purpose, we have first plotted in Figs. 8 (a) and 8(b) the real and imaginary parts of the effective permittivity for spherical inclusions and different values of $\varepsilon_{1}^{\prime}$ and $\varepsilon_{1}^{\prime \prime}$. It may be seen that the major effect of shape anisotropy is a strong increase of $\varepsilon^{\prime \prime}$. This is demonstrated by a comparison of Figs. 8(c) and 8(d). From Figs. 8(c) and $8(\mathrm{~d})$, one can see that pronounced changes (up to a factor of 10) in the imaginary part of the effective permittivity when the inclusions have complex permittivity extremely different from the host medium.

\section{Ellipsoids}

In a similar way, we carried out additional calculations with ellipsoidal inclusions. The results are shown in Figs. $8(\mathrm{e})$ and $8(\mathrm{f})$. The earlier conclusion that we drew for rodlike inclusions is valid here as well.

\section{DISCUSSION}

Collectively, the data reported here demonstrate that the shape anisotropy of inclusions in dielectric heterostructures dramatically affect the effective complex permittivity. Remarkably, we found that the square law dependence of the conduction threshold versus the aspect ratio applies for both rodlike and ellipsoidal inclusions, over a wide range of aspect ratios. Based on the preceding observations it is important to make a number of qualifying remarks. First, the effective permittivity of dielectric heterostructures composed of inclusions having shape anisotropy can be an extremely sharp function of volume fraction of inclusions, depending on the aspect ratio, and the orientation and the conductivity of the inclusions. The results we have obtained are consistent with previous observations made by several groups. ${ }^{13-16,20}$ Second, one of the most interesting conclusions which arises from the results of Sec. III is that the transition from insulating to conducting behavior is critical, exhibiting scaling laws. There are indications of unconventional critical exponents from power-law volume fraction dependences of the permittivity. A study of the scaling laws reveals lessons for how to make dielectric heterostructures having a low conduction threshold. Third, our results illustrate the importance of properly describing the alignment and contact of inclusions in the matrix, a result that opens up interesting possibilities for the future analysis of experimental data.

Shape anisotropy and spatial orientation in the dielectric properties of heterostructures are of considerable interest, especially in the light of the suggestion that the conduction threshold is related to the actual area of contacts between the inclusions. ${ }^{3}$ The essential point is that the different behavior between isotropic and anisotropic inclusions must reflect the surface area of the inclusions. What general statements can we now make about the connection between conduction threshold and contact? The answer can be guessed on symmetry grounds. To explain, we may state matters as follows: Spherical shape provides a minimum of surface area for a given volume fraction and a fixed number of inclusions. Similarly, elongated shapes, which deviate from spherical, provide less surface area for a given number and volume fraction of inclusions. Consequently, spherical inclusions must occupy a higher volume fraction before guaranteeing contact for the existence of a continuous path that crosses the sample. We may observe that, for inclusions having anisotropic shapes, the full rotational symmetry of the problem is broken at the critical point, a unique preferred direction is picked with different critical exponents than the isotropic problem: this is apparent from a comparison of $s^{*}$ and $t^{*}$ in Table I. As this work indicates, the sharp increase in the permittivity when plotted as a function of volume filling fraction characterizes higher electric multipole interactions because all of the inclusions contact at once. ${ }^{9}$ We note also that the values of $f^{*}$ are consistent with analytical results from theoretical predictions based on the concept of excluded volume associated with three-dimensional objects. ${ }^{16}$ The excluded volume is much larger than the actual volume for extended shapes, such as rods and ellipsoids. Then, the resulting $f^{*}$ may be lower than for an array of spheres. For spheres, the actual and excluded volumes are the same. Equally significant, we observed a strong orientational effect on the permittivity. This again indicates that the more extended shape which deviates from spherical provides what is needed for contact: they are characterized by more favorable depolarization factors.

Many authors, based on both experimental and theoretical work, have discussed the deviation from universality of the $s$ and $t$ exponents. ${ }^{17,19,20,36}$ For example, Chen and Johnson obtained a lower $f_{c}(0.075)$ and a higher $t$ (3.1) for filamentary nickel than for nodular nickel $\left(f_{c}=0.265\right.$ and $t$ $=2.2$ ) both in polypropylene. ${ }^{19}$ Lee et al. argued that one can only expect universal exponents if the local microstructure is isotropic and contains only short-range correlations. ${ }^{37}$ It is equally true, however, that physical mechanisms other than contact are also involved if we are not dealing with an 

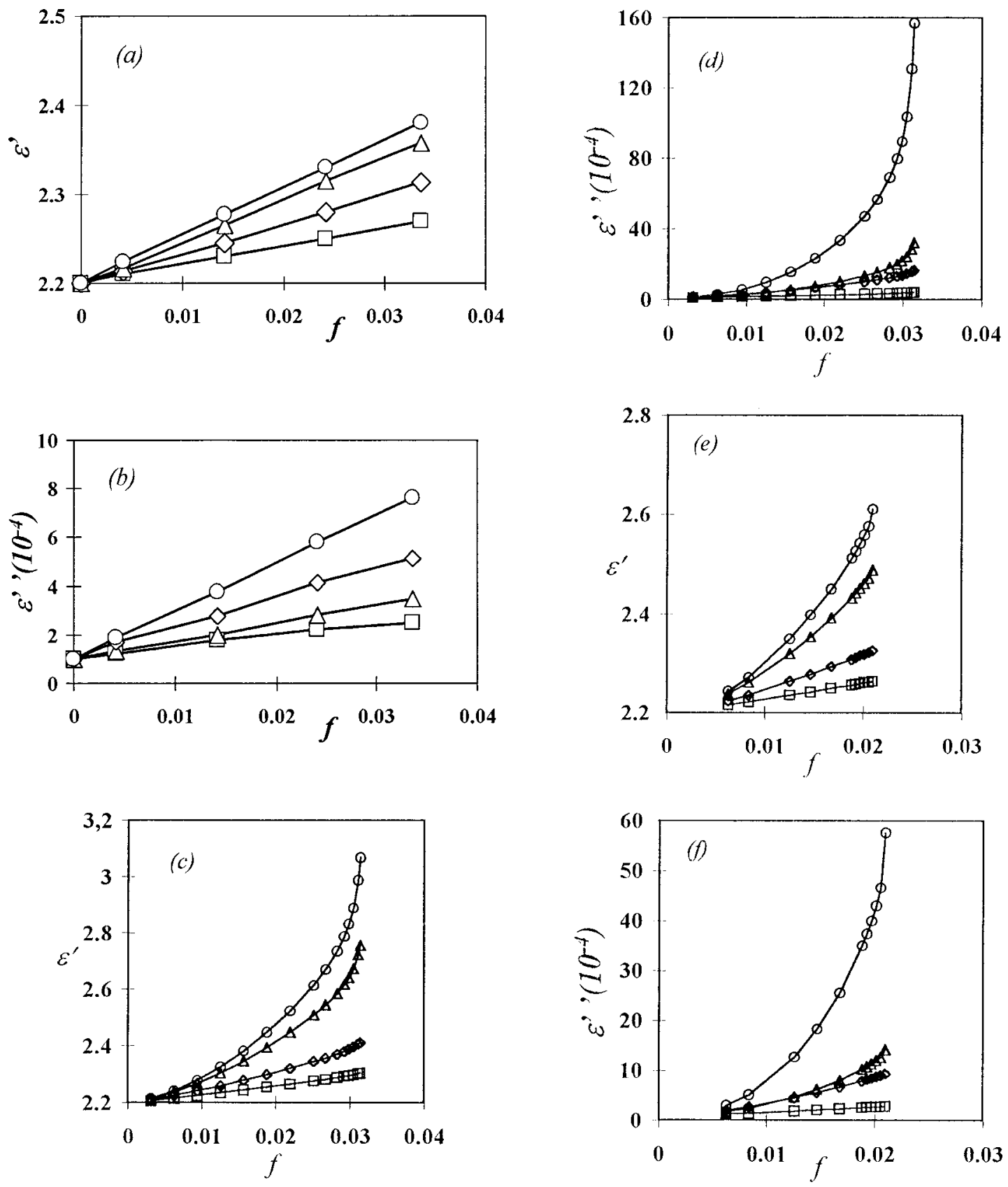

FIG. 8. (a) Real part of the effective permittivity $\varepsilon^{\prime}$ is shown as a function of the volume fraction $f$ of the inclusion phase. Spherical inclusions (permittivity $\left.\varepsilon_{1}=\varepsilon_{1}^{\prime}-i \varepsilon_{1}^{\prime \prime}\right)$ are placed in a host matrix material of permittivity $\varepsilon_{2}=2.2-i 10^{-4}$. Simple-cubic lattice configuration. Symbols are: $(\square) \varepsilon_{1}=5.5-i 10^{-2}$, $(\diamond) \varepsilon_{1}=9-i 510^{-2},(\triangle) \varepsilon_{1}=20-i 10^{-1}$, and $(\bigcirc) \varepsilon_{1}=30-i 0.5$. The solid lines are guides to the eye. (b) Same as in (a) for the imaginary part of the effective permittivity $\varepsilon^{\prime \prime}$. (c) Same as in (a) for the rodlike inclusions. $r=0.10$. The direction of the applied electric field is parallel to the rod axis. (d) Same as in (c) for the imaginary part of the effective permittivity $\varepsilon^{\prime \prime}$. (e) Same as in (a) for the ellipsoidal inclusions. $a=b=0.10$. The direction of the applied electric field is parallel to the principal symmetry axis. (f) Same as in (e) for the imaginary part of the effective permittivity $\varepsilon^{\prime \prime}$.

idealized computer model but with real engineering materials. Many questions still remain as to what factors dominate, particularly when the interfacial contact is mediated by adsorption zones that introduce parameters such as adhesive interactions at contact, tunneling phenomena, and partial wetting. This lack of knowledge about structures has limited an understanding of the physical properties of these materials.

\section{CONCLUDING REMARKS}

We now summarize our findings and suggest some avenues for future research. We have performed ab initio calculations of the effects of shape anisotropy and orientation of inclusions in periodic (deterministic) arrangements on the complex permittivity of 1-3 composites, in the quasistatic limit. In the work reported here, we focused on two specific examples: rodlike and ellipsoidal inclusions. Besides being 
important in their own right, these effects have considerable interest and play a key role in a diverse range of problems in engineering areas, as well as in biological processes. The ability to systematically control the properties of dielectric heterostructures by variation of size and shape is an important development with many implications for how these materials should be processed and assembled. The conduction threshold of a two-component heterostructure is an important quantity which can give useful information on the shape anisotropy of the materials. It seems reasonable to conclude that a low threshold for the onset of conduction can be achieved in practice by tuning the geometrical parameters of the inclusions. From these results, we find that the close packing condition accounts well for the aspect ratio dependence of conduction threshold. Returning to the title question, we emphasize that the effective permittivity of systems containing isotropic and anisotropic shapes of inclusions differ markedly, reflecting the geometrical connectivity of this spatial arrangement. This point is made more clear by appeal to Figs. 2(a) and 2(b), Figs. 4(a) and 4(b), and Fig. 8. Using numerical evidence we have argued for the appropriateness of a critical behavior to describe the sharp increase of the permittivity when the filler concentration $f$ becomes higher than a value $f^{*}$. In fact, one can sweep across broad ranges of permittivity and conductivity with only modest changes in the aspect ratio by working "close" to the conduction threshold concentration. We have mentioned that this scaledependent behavior has been rationalized by arguing that inclusions having higher surface area per volume favor easy formation of conductive networks at lower concentration of inclusions and exhibit a lower conduction threshold. Additionally, this work has shown that the two static exponents $s^{*}$ and $t^{*}$ are different from the presumably universality values $s$ and $t$ derived from the standard (geometrical) percolation model. It should also be noted that the electrical response of the composites is affected by the manner in which the field is applied: the transverse conductivity is smaller than the axial conductivity.

The continued miniaturization of electronic devices is leading us into a realm of nanostructures, which exhibit electronic and optical properties. The technological utility of ultrasmall cylinders is a strong function of their mechanical robustness and electromagnetic properties. Metallized tubules offer an attractive geometry for microwave applications. ${ }^{34}$ Moreover, because of this large aspect ratio, fiber-filled composites at low volume fractions can have elastic moduli orders of magnitude larger than that of composites containing carbon black aggregates at the same volume fraction. The controlled assembly of materials with patterned structural features on a given length scale is a rapidly expanding area in materials science, e.g., Ajayan et al. described a technique that produces aligned arrays of carbon nanotubes. ${ }^{38}$ One of the greatest challenges involved in the development of advanced dielectric heterostructures is accurate characterization of the size, shape, and orientation of individual inclusions. This challenge must be met in order to optimize electromagnetic shielding properties. As was pointed out by Sau et al., short carbon fibers filled compos- ites are more effective than particulate filled composites in providing good shielding from electromagnetic interference. ${ }^{39}$

A systematic test with experiments has not yet been carried out, although such a comparison is necessary for advancing our understanding of these fascinating materials. In large part, the present work was motivated by our interest in composite materials, in particular those involving carbon fiber and carbon black aggregates inclusions. ${ }^{40-43}$ Such materials are predicted to be rich in physics exhibiting properties such as diverse as magnetoresistance, hopping, and tunneling phenomena. ${ }^{43,44}$ Several important aspects of the dielectric properties of heterostructures were not addressed in this study. Some of these issues can be addressed numerically, and we plan to continue these studies to explore this issue when the mixture geometry is precisely specified. Hopefully, experiment can also play a role. Already, important experimental contributions have been made concerning different materials by a number of groups. A test of these numerical results requires a highly purified sample with careful rod length distribution. A number of important issues remain to be addressed as far as this simulation work is concerned. First, while the numerical calculations presented here were derived for regularly shaped, sized, and periodically distributed inclusions in a simple cubic lattice, the formalism on which they are based is quite versatile and can be extended to other situations, e.g., the more general problem of the anisotropic and tensorial conductive behavior of dielectric heterostructures. Yet, another dimension of complexity is added by the fundamental question of randomness. The effective permittivity of random heterostructures may be calculated only approximately, even for monodisperse spheres. ${ }^{26}$ For random systems, we expect that two factors govern the contact between inclusions: geometrical and statistical. Geometrically, the number of contact points per inclusion is related to the surface area of the inclusion; statistically the contacts involving a given component increase with the increase in the amount of that component. Even though an acceptable description of the complex threedimensional structure with the interface surfaces oriented in random directions with respect to the applied electric field has been reported, it does not provide a satisfactory description of the dielectric properties of real heterostructures for which specific interactions between the inclusions and the host matrix need be taken into account. Moreover, in (random) composites containing complex-shaped constituents, an exact calculation of higher multipole interactions is intractable. Evidently, the richness of the polarization phenomena for heterostructures with inclusions having irregular geometries poses a severe challenge to any complete theoretical understanding. Work is in progress in that direction but is computationally very demanding. A more detailed knowledge of the scattering phenomena could also be helpful in the design of periodic dielectric systems in the context of photonic bang gap structures. ${ }^{45}$ 


\section{ACKNOWLEDGMENTS}

Laboratoire d'Électronique et Systèmes de Télecommunications is Unité Mixte de Recherche CNRS 6616 and Center de Génie Électrique de Lyon is Unité Mixte de Recherche CNRS 5005.

${ }^{1}$ Progress in Electromagnetics Research: Dielectric Properties of Heterogenous Materials, edited by A. Priou (Elsevier, New York, 1992) and references therein. The number of papers devoted to characterizing the electromagnetic properties of heterostructures is so large that no attempt to reference them will be made here.

${ }^{2}$ A. Boudida, A. Beroual, and C. Brosseau, J. Appl. Phys. 83, 425 (1998).

${ }^{3}$ A. Beroual, C. Brosseau, and A. Boudida, J. Phys. D: Appl. Phys. 33, 1969 (2000).

${ }^{4}$ J. P. Clerc, G. Giraud, J. M. Laugier, and J. M. Luck, Adv. Phys. 39, 191 (1990).

${ }^{5}$ D. Stauffer and A. Aharony, Introduction to Percolation Theory, 2nd ed. (Taylor \& Francis, London, 1992). See also R. Zallen, The Physics of Amorphous Solids (Wiley, New York, 1983).

${ }^{6}$ S. Kirpatrick, Rev. Mod. Phys. 45, 574 (1973). See also S. Redner and H. G. Stanley, J. Phys. A 12, 1267 (1979); H. Ikeda, Prog. Theor. Phys. 61, 842 (1979), J. P. Troadec, D. Bideau, and E. Guyon, J. Phys. C 14, 4807 (1981), and C.-W. Nan, Prog. Mater. Sci. 37, 1 (1993).

${ }^{7}$ In a two-component composite material, the different combinations of component connectivity are indicated using two digits denoting the conductivity of the filler and that of the matrix respectively in this order. See R. E. Newham, D. P. Skinner, and L. E. Cross, Mater. Res. Bull. 13, 525 (1978). Fibers implanted in a matrix and oriented across one of the dimensions constitute a 1-3 composite.

${ }^{8}$ D. S. McLachlan, M. Blaszkiewicz, and R. E. Newnham, J. Am. Ceram. Soc. 73, 2187 (1990).

${ }^{9}$ W. T. Doyle, J. Appl. Phys. 78, 6165 (1995). See also W. T. Doyle and I. S. Jacobs, Phys. Rev. B 42, 9319 (1990).

${ }^{10}$ R. Landauer, in Electric Transport and Optical Properties of Inhomogeneous Media, edited by J. C. Garland and D. B. Tanner, AIP Conf. Proc. No. 40 (American Institute of Physics, New York, 1978), p. 2.

${ }^{11}$ M. Sahimi, Applications of Percolation Theory (Taylor and Francis, London, 1994)

${ }^{12}$ P. C. Sturman and R. L. McCullough, J. Appl. Phys. 72, 2883 (1992).

${ }^{13}$ F. Carmona, F. Barreau, P. Delhaes, and R. Conet, J. Phys. (France) Lett. 41, L531 (1980). See also F. Carmona, P. Delhaes, F. Barreau, D. Ordiera, R. Canet, and L. Lafeychine, Rev. Chim. Miner. 18, 498 (1981).

${ }^{14}$ A. N. Lagarkov and A. K. Sarychev, Phys. Rev. B 53, 6318 (1999). See also A. N. Lagarkov, S. M. Matytsin, K. N. Rozanov, and A. K. Sarychev, Physica A 241, 58 (1997), and A. N. Lagarkov, S. M. Matytsin, K. N. Rozanov, and A. K. Sarychev, J. Appl. Phys. 84, 3806 (1998).

${ }^{15}$ The $r / 1$ dependence of the percolation threshold concentration in fiber filled composites was also discussed by several independent groups, raising a legitimate doubt on the general validity of the square law dependence for intermediate values of $r / 1$. Thus, there is some uncertainty over this issue. See J. Boissonade, F. Barreau, and F. Carmona, J. Phys. A 16, 2777 (1983); A. Celzard, E. McRae, C. Deleuze, M. Dufort, G. Furdin, and J. F. Marêché, Phys. Rev. B 53, 6209 (1998), and E. J. Garboczi, K. A. Snyder, J. F. Douglas, and M. F. Thorpe, Phys. Rev. E 52, 819 (1995).

${ }^{16}$ I. Balberg, C. H. Anderson, S. Alexander, and N. Wagner, Phys. Rev. B 30, 3933 (1984). See also I. Balberg, N. Binenbaum, and N. Wagner, Phys. Rev. Lett. 52, 1465 (1984), and I. Balberg, Philos. Mag. B 56, 991 (1987).

${ }^{17}$ F. Carmona and A. El Amarti, Phys. Rev. B 35, 3284 (1987).

${ }^{18}$ S. L. Browning, J. Lodge, R. R. Price, J. Schelleng, P. E. Schoen, and Z. Zabetakis, J. Appl. Phys. 84, 6109 (1998).

${ }^{19}$ I. G. Chen and W. B. Johnson, J. Mater. Sci. 26, 1565 (1991).

${ }^{20}$ E. J. Garboczi, K. A. Snyder, J. F. Douglas, and M. F. Thorpe, Phys. Rev. E 52, 819 (1995).

${ }^{21}$ B. T. Shklovskii, Phys. Status Solidi B 85, K111 (1978).

${ }^{22}$ K. J. Singh, R. Singh, and D. R. Chaudhary, J. Phys. D 31, 1681 (1998).
${ }^{23}$ R. E. Diaz, W. M. Merrill, and N. G. Alexopoulos, J. Appl. Phys. 84, 6815 (1998).

${ }^{24}$ B. Sareni, L. Krahenbühl, A. Beroual, and C. Brosseau, J. Appl. Phys. 80, 4560 (1996)

${ }^{25}$ B. Sareni, L. Krahenbühl, A. Beroual, and C. Brosseau, J. Appl. Phys. 80, 1688 (1996)

${ }^{26}$ B. Sareni, L. Krahenbühl, A. Beroual, and C. Brosseau, J. Appl. Phys. 81, 2375 (1997).

${ }^{27}$ J. P. Calame, A. Birman, Y. Carmel, D. Gershon, B. Levush, A. A. Sorokin, V. E. Semenov, D. Dadon, L. P. Martin, and M. Rosen, J. Phys. D 80, 3992 (1996)

${ }^{28}$ P. K. Ghosh and M. E. Azimi, IEEE Trans. Dielectr. Electr. Insul. 1, 975 (1994).

${ }^{29}$ R. T. Coverdale, E. J. Garboczi, and H. M. Jennings, Comput. Mater. Sci. 3, 465 (1995). See also R. T. Coverdale, B. Christensen, T. O. Masov, H. M. Jennings, and E. J. Garboczi, J. Mater. Sci. 29, 4984 (1994).

${ }^{30}$ A. P. Roberts and M. A. Knackstedt, Phys. Rev. E 54, 2313 (1996).

${ }^{31}$ D. Cule and S. Torquato, J. Appl. Phys. 86, 3428 (1999).

${ }^{32}$ C. Brosseau and A. Beroual, Eur. Phys. J.: Appl. Phys. 6, 23 (1999).

${ }^{33}$ G. K. Batchelor and R. W. O'Brien, Proc. R. Soc. London, Ser. A 355, 313 (1977). For a closely packed set of spheres randomly arranged in a matrix, Batchelor and O'Brien used lubrication theory and obtained for perfectly conducting spheres a logarithmic divergence of the effective thermal (or electrical) conductivity of the material, i.e., in our notation $s^{*}=0$. However, this result is by no means conclusive since it takes into account only dipolar interactions. See also for a more recent approach D. J. Bergman, Phys. Rev. B 39, 4598 (1989). Here, the author has derived analytical expressions for the divergence of $\varepsilon^{\prime}$ and $\varepsilon^{\prime \prime}$ of cylindrical inclusions at the critical point. In this approach, the critical exponents $s^{*}$ $=1$ and $t^{*}=1$ are mean-field exponents. However, as has been widely discussed in the literature, the predictions of mean-field theories often contradict many of the experimental data on random composite materials because these theories do not describe accurately the actual multipole interactions at the critical point.

${ }^{34}$ A. R. Blythe, Electrical Properties of Polymers (Cambridge University Press, Cambridge, 1980).

${ }^{35}$ Y. Agari, A. Veda, and S. Nagai, J. Appl. Polym. Sci. 52, 1223 (1994).

${ }^{36}$ See, for example, C. Brosseau, J. Appl. Phys. 75, 672 (1992) and references therein.

${ }^{37}$ S. Lee, Y. Song, T. Noh, and J. R. Gaines, Phys. Rev. B 34, 6719 (1986).

${ }^{38}$ P. M. Ajayan, O. Stephan, C. Colliex, and D. Trauth, Science 265, 1212 (1994). Carbon nanotubes are tubular structures that are typically nanometers in diameter and many microns in length.

${ }^{39}$ K. P. Sau, D. Khastgir, and T. K. Chaki, Angew. Makromol. Chem. 258, 11 (1998). See also K. P. Sau, T. K. Chaki, A. Chakraborty, and D. Khastgir, Plast. Rubb. Compos. Proc. Appl. 26, 291 (1997).

${ }^{40}$ For a survey of recent investigations, see Carbon Black, Science, and Technology, edited by J. B. Donnet, R. C. Bansal, and M. J. Wang (Marcel Dekker, New York, 1993). See also A. I. Medalia, Rubber Chem. Technol. 51, 437 (1978).

${ }^{41}$ C. Brosseau, F. Boulic, C. Bourbigot, P. Queffelec, Y. Le Mest, J. Loaëc, and A. Beroual, J. Appl. Phys. 81, 882 (1997). See also F. Boulic, C. Brosseau, Y. Le Mest, J. Loaëc, and F. Carmona, J. Phys. D 31, 1904 (1998), and C. Brosseau, P. Molinié, F. Boulic, and F. Carmona (unpublished).

${ }^{42}$ Short-Fibre-Polymer Composites, edited by S. K. De and J. R. White (Technomie Publishing Co., Inc., New York, 1997).

${ }^{43}$ F. Carmona, Physica A 157, 461 (1989). It should be emphasized that in conductive polymer composites, the filler volume fraction at which a network of touching particles is formed is not an invariant but depends strongly on the processing conditions.

${ }^{44}$ R. Schueler, J. Petermann, K. Schulte, and H. P. Wentzel, J. Appl. Polym. Sci. 63, 1741 (1997). See also J. Sandler, M. S. P. Shaffer, T. Prasse, W. Bauhofer, K. Schulte, and A. H. Windle, Polymer 40, 5967 (1999).

${ }^{45}$ Photonic Bang Gaps and Localization, edited by C. M. Soukoulis (Plenum, New York, 1993). 